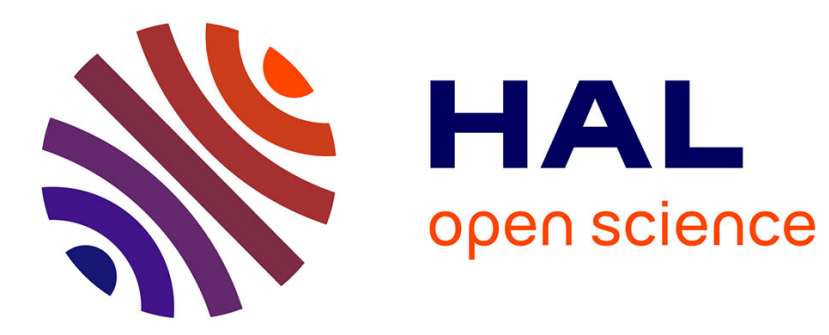

\title{
Stimulated Cherenkov Radiation of Plasma Waves and Plasma Turbulence
}

A. Rukhadze, K. Sarksyan, N. Skvortsova

\section{To cite this version:}

A. Rukhadze, K. Sarksyan, N. Skvortsova. Stimulated Cherenkov Radiation of Plasma Waves and Plasma Turbulence. Journal de Physique IV Proceedings, 1995, 05 (C6), pp.C6-53-C6-59. 10.1051/jp4:1995610 . jpa-00253973

\section{HAL Id: jpa-00253973 https://hal.science/jpa-00253973}

Submitted on 1 Jan 1995

HAL is a multi-disciplinary open access archive for the deposit and dissemination of scientific research documents, whether they are published or not. The documents may come from teaching and research institutions in France or abroad, or from public or private research centers.
L'archive ouverte pluridisciplinaire HAL, est destinée au dépôt et à la diffusion de documents scientifiques de niveau recherche, publiés ou non, émanant des établissements d'enseignement et de recherche français ou étrangers, des laboratoires publics ou privés. 


\title{
Stimulated Cherenkov Radiation of Plasma Waves and Plasma Turbulence
}

\author{
A.A. Rukhadze, K.A. Sarksyan and N.N. Skvortsova \\ General Physics Institute of Russian Academy of Sciences, 117942 Moscow, Russia
}

\section{General Ideology}

Stimulated Cherenkov Radiation of plasma waves represents a type of radiative instability of the plasma-beam system and current driven plasma. If the system is near the threshold of instability then the coherent practically monochromatic plasma waves are created, whereas when the system is significantly above the threshold then the wide-band and chaotic radiation is excited.

Bellow the results of theoretical, numerical and experimental investigations of stimulated Cherenkov radiation of the dense electron streams in a bounded magnetized plasma carried on in GPI are presented. It will be considered two examples: stimulated radiation of microwaves by Relativistic Electron Beams (REB) in a bounded plasma and stimulated radiation of ion-sound waves in a current driven plasma.

a) Stimulated radiation of microwaves by REB in a bounded plasma is a basic phenomenon for the relativistic plasma electronics, for the high power plasma sources of coherent microwave radiation. Just in this case the instability near the threshold occurs to be long wave length and therefore the practically monochromatic and coherent waves excitation with high efficiency of radiation takes place. However if the beam-plasma system is significantly above the threshold then the spectrum of excited waves becomes wide-band, the electric field of plasma oscillations occurs to be almost potential and the output radiation quickly decreases. In this case one can say that the Langmuir turbulence is excited in a plasma.

b) Stimulated radiation of ion-sound waves in a current driven non isothermal plasma is caused by the motion of hot electrons through the cold ions. This phenomenon is well-known also as ion-sound instability of a current driven plasma and it is very important for thermonuclear devices. This instability, in contrary to the considered above, near the threshold occurs to be short wave length. Therefore even near the threshold excitation of sufficiently wide-band and chaotic oscillations takes place, or in other words the ion-sound instability of current driven plasma in all cases is accompanied by developing of a turbulence.

\section{Stimulated Cherenkov Radiation of High- Frequency Waves. Langmuir Turbulence}

The phenomenon was investigated in a concrete experimental device TEREK-II [1]. The hollow REB $\left(r_{b}=\right.$ $0,6 \mathrm{~cm}, \Delta_{b} \leq 1 \mathrm{~mm}, E_{b} \leq 700 \mathrm{keV}, \tau_{b} \simeq$ $100 \mathrm{~ns}, I_{b} \leq 10 \mathrm{kA}$ ), emitted by the cathode and accelerated in the diode gap, propagates in the drift chamber and impinges on the collector. The plasma layer $\left(n_{p} \leq 10^{14} \mathrm{~cm}^{-3}, r_{p}=\right.$ 0,$\left.7 ; 0,8 ; 0,9 \mathrm{~cm}, \Delta_{p} \leq 1 \mathrm{~mm}\right)$ is crated by the beam from thermocathode $(E \simeq 600 \mathrm{eV}, I \simeq 100 \mathrm{~A}, \mathrm{Xe}, P \simeq$ $\left.2 * 10^{-3} \mathrm{mmHg}, \tau_{d} \simeq 15 m k s\right)$. The $P, f, \triangle f / f$ dependences on $n_{p}$ are mea- 
sured. The magnetic field $B_{0} \simeq 2,5 T L$.

Instability threshold in the long wave length region is

$$
\begin{aligned}
& \omega_{p}^{2}>\omega_{p t h}^{2}=k_{\perp m i n}^{2} u^{2} \gamma^{2}, \\
& k_{\perp l}^{2}=\left\{\begin{array}{cl}
\frac{1}{r_{p} \Delta_{p}} l n^{-1} R / r_{p}, & l=0, \\
\frac{2}{r_{p} \Delta_{p}}\left(1-r_{p}^{2 l} / R^{2 l}\right)^{-1}, & l \neq 0,
\end{array}\right. \\
& \gamma^{2}=\left(1-\frac{u^{2}}{c^{2}}\right)^{-1}
\end{aligned}
$$

where $\omega_{p}$ - is the plasma frequency. The frequency and growth rate of radiated waves when $\omega_{p}^{2}>\omega_{p t h}^{2}$ :

$$
\begin{aligned}
& \omega \approx k_{\|} \approx \sqrt{\omega_{p}^{2}-k_{\perp l}^{2} u^{2} \gamma^{2}} \\
& \delta \omega=\delta k_{\|} u= \\
& \left\{\begin{array}{c}
\sqrt{3} / 2\left(\beta^{2} \frac{\omega \omega_{b}^{2}}{\gamma^{3}}\right)^{1 / 3}>\omega_{b} \gamma^{-3 / 2}, \\
\sqrt{1} / 2\left(\beta^{2} \frac{\omega \omega_{b}}{2 \gamma^{3 / 2}}\right)^{1 / 2}<\omega_{b} \gamma^{-3 / 2},
\end{array}\right.
\end{aligned}
$$

where $\beta<1$ depends from the ratio $r_{b} / r_{p}$. If

$$
\delta \omega>\frac{u}{L} \ln 3 \gamma^{2}
$$

or the beam current exceeds critical one $I_{e r}$ then the system is working as oscillator, $L$ - is the length of system. If $\omega_{p}^{2} \geq \omega_{p t h}^{2}$ the spectrum of waves is narrow and when $\omega_{p}^{2} \gg \omega_{p t h}^{2}$ it becomes wider; finally the turbulence arises.

From the Fig. 1 it is seen that the stimulated radiation arises when $n_{p}>$ $n_{p t h} \simeq 2 * 10^{12} \mathrm{~cm}^{-3}$. When $n_{p} / n_{p t h}$ increases the frequency band of waves increases also. However when $n_{p} / n_{p t h} \gg$ 1 it occurs to be narrow again, because in this case the frequencies of all modes of oscillations tend to $\omega_{p}$.

The two solid curves in the Fig.2 are drawn by using the results showed in the Fig.1. The vertical distances between them determine the excited waves frequency bands. The arrows show the experimental spectrum limits obtained from the dates in the Fig.8.

The nonlinear theory is mainly based on the numerical simulations. We used the $3 \mathrm{D}^{*} 3 \mathrm{D}$ KARAT code [2] for solving the Maxwell-Vlasov equations. Bellow we will give only the results.

In the Fig. 3 the dependence of radiation efficiency on the beam current is shown when $n_{p} \geq n_{p t h}$ and single mode wave excitation takes place. The maxi-

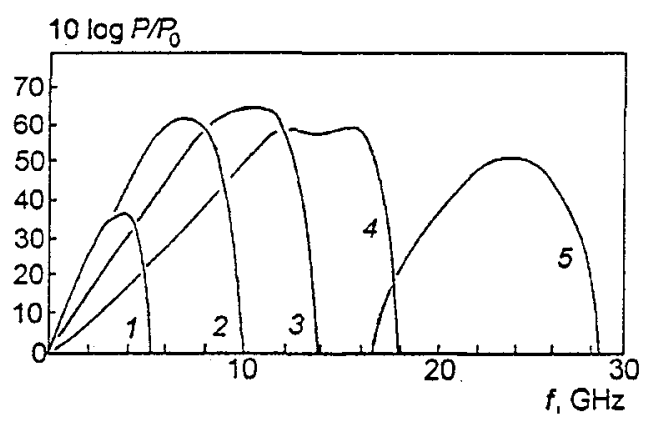

Figure 1: Power gain dependencies on the wave frequency for several values of the plasma density, calculated numerically: (1) $3.3 * 10^{12}$, (2) $7.5 * 10^{12},(3)$ $1.33 * 10^{13}$, (4) $2.1 * 10^{13},(5) 4.1 * 10^{13}$.

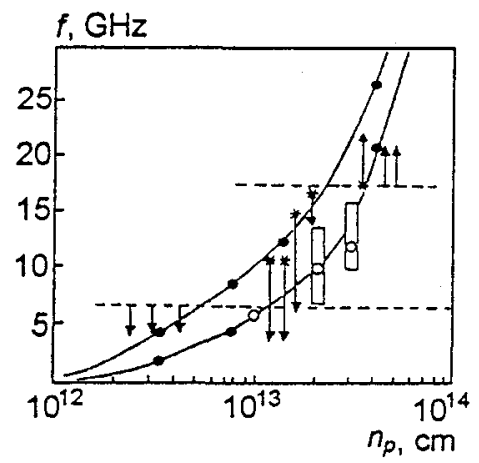

Figure 2: Microwave radiation spectrum dependencies on the plasma density; comparison of experimental results with numerical calculations.

mal efficiency is reached when $I_{b} / I_{c r} \simeq$ 3. In the region $I_{c r}<I_{b}<3 I_{e r}$ it increases as $I_{b}^{1 / 3}$ and when $I_{b}>3 I_{e r}$ it droops as $I_{b}^{-1}$.

In the Fig.4 the dependence of ra- 
diation efficiency on the dimensionless time $\tau$ is presented for the different values of parameter

$$
\xi=\frac{\omega}{u} L\left(\frac{1}{2} \frac{\omega_{b}^{2} \gamma^{-7}}{k_{\perp}^{2} u^{2}}\right)^{1 / 3}
$$

When $\xi>\xi_{0} \approx 2,55$ which corresponds to the condition $\operatorname{Im} \delta \omega=0$, the wave excitation takes place and our system becomes as oscillator. When $\xi<\xi_{0}$ the waves are damping. The curve 1 (damping) in the Fig.4a corresponds $\xi_{1} \simeq 2,41$, for curves 3 and 4 respectively $\xi_{2} \simeq 2,65$ and $\xi_{3} \simeq 2,79$, or $\xi_{2,3}>\xi_{0}$ and we see the waves increasing, but the growth rate are small and the times of waves growing occur to be sufficiently large. Finally $\xi_{3} \simeq 3,03$

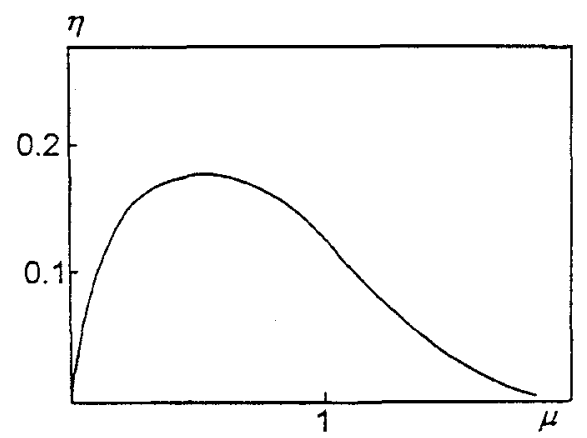

Figure 3: Conversion efficiency $\eta$ dependence on $\mu=2 \gamma^{2} \delta \omega / \omega$.

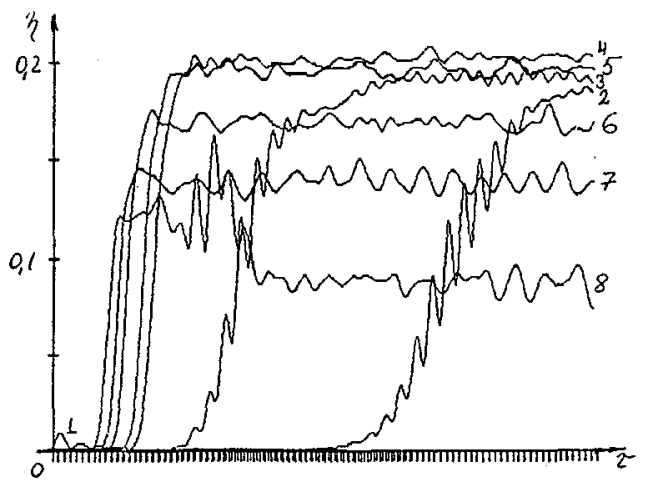

Figure 4: Dependence of radiation efficiency $\eta$ on dimensionless time $\tau$. is optimal - for this value of $\xi$ just at $z=L$ the beams electrons capturing takes place and therefore the efficiency $\eta$ occurs to be maximal. The curves 5 8 in the Fig. 4 correspond to $\xi>3,03$. The radiation efficiencies begin drop and at $\xi_{8} \simeq 4$ radiation efficiency becomes very small. At the same time the excited waves become chaotic and their average frequency tends to the plasma frequency. One can said that it arises in the system Langmuir turbulence.

The phenomenon of the waves turbulization is seen also from the phase diagrams, Rea $=F(\operatorname{Ima})$, which are presented in the Fig.5a,b,c,d for different values of $\xi[3]$. The picture 5 a corresponds to $\xi_{1} \simeq 2,41$, when all the oscillation are damping. The picture $5 \mathrm{~b}$ corresponds to $\xi_{3}=3,03$ - optimal wave excitation, it is regular, or, no turbulence. The picture $5 \mathrm{c}$ corresponds to $\xi_{5}=3,5$ - the waves become non regular and chaotic, and when $\xi_{8}=4$ (picture $5 b$ ) we see full chaotization, it corresponds to the strong Langmuir turbulence.

All experimental measurement were carried on the device TEREK-II [1]. There were measured: plasma density dependence of the radiation power (measured by a calorimeter, Fig.6, $I_{b} / I_{c r} \simeq 3$ ); plasma density dependence of the radiation power for dif-
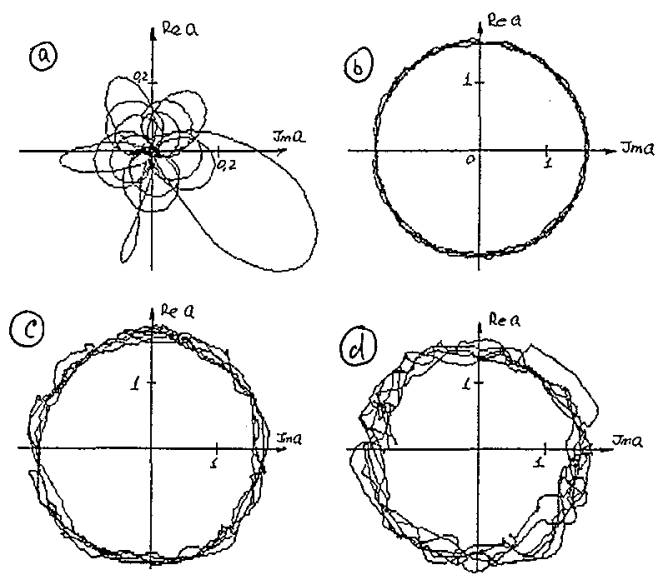

Figure 5: Phase diagrams of waves. 


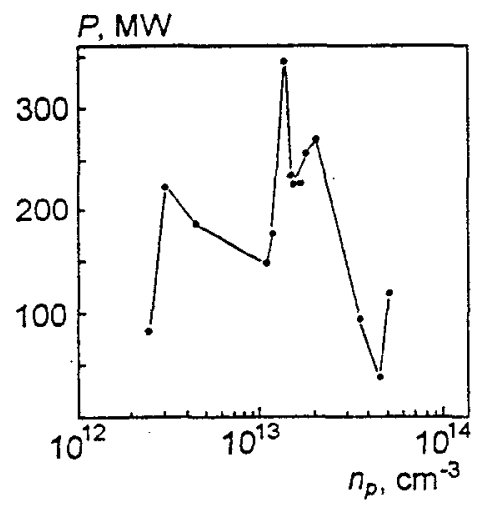

Figure 6: Radiation power dependence on the plasma density, measured by the energy calorimeter.

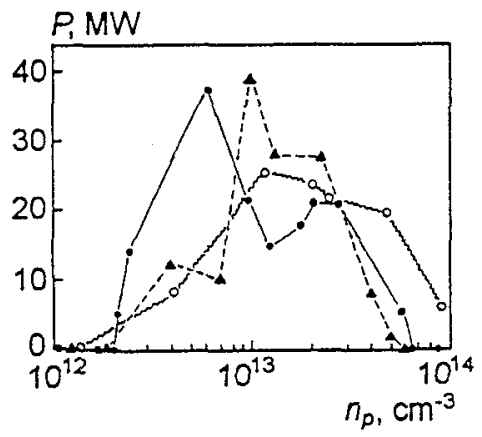

Figure 7: Radiation power dependencies on the plasma for $L=30 \mathrm{~cm}$ and several values of the beam current: the triangles correspond to $I_{b}=2 k A$, filled circles and open circles correspond to $I_{b}=3 k A$ and $I_{b}=4 k A$, respectively.

ferent beam currents (Fig.7, $I_{b} / I_{c r} \simeq$ $2, I_{b} / I_{c r} \simeq 3$ and $I_{b} / I_{c r} \simeq 4$ ); radiation spectra for several shots and for different plasma densities (Fig.8 and Fig.2). The experimental dates are in a good agreements with the theoretical results showed in the figures $2,3,4$ and 5. Besides, the wide band spectrum of radiation observed when $n_{p} / n_{p t h} \gg 1$ corresponds to the Langmuir turbulence showed in the Fig.5.

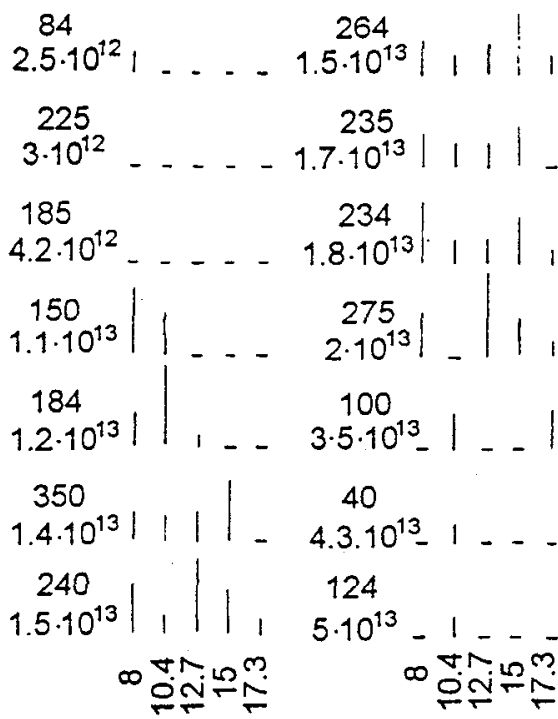

Figure 8: Experimental results of spectral measurements of microwave radiation.

\section{Low-frequency turbu- lence}

The experiments in the lowfrequency range of plasma waves (ionsound turbulence) were carried out in model linear device TAU-1 [4]. The plasma was produced by the electron beam $\left(U_{b}=-50 \mathrm{~V}--150 \mathrm{~V}, I_{b}=50-\right.$ $150 \mathrm{~mA}$ ). We used argon as a working gas at pressure of $3 * 10^{-4}$ torr, the intensity of the magnetic field was $H=$ $0.4-0.6 T$. Plasma density on the axis - $n_{e}(0)=7 * 10^{9}-4 * 10^{10} \mathrm{~cm}^{-3}$, electron temperature $-T_{e}(0)=5-7 e V$, ion temperature $-T_{i}(0)=0.1 T_{e}(0)$.

We used the probe technique to measure the main plasma parameters and the low-frequency fluctuations of plasma density. Fluctuation signals were analyzed by different numerical methods of spectral estimations. We calculated statistical moments of signals, theirs probability distributions, and time and space correlation functions to provide information necessary for this analysis. The frequency spectra were obtained by using a fast Fourier 
transform (FFT) routine with the preliminary choice of appropriate windows. The maximum entropy method was used to test results obtained with FFT. We calculated bicoherence function to study three-wave coupling in investigated turbulence. The limitation of these methods is due to the turbulent character of the probe signal. Thus the additional smoothing and averaging of the output data is necessary to get statistically steady result. Two types of plasma low-frequency wave spectra were observed in the experiment. The first spectrum was the discrete frequency spectrum of drift ionsound waves in frequency range of $20-$ $100 k H z$. These waves were excited by the drift instability in the region of the lateral plasma gradient [5]. The turbulent ion-sound waves $\left(\omega_{s}\right)$ with the continuum spectrum extended up to the ion langmuir frequency $\left(\omega_{L i}\right)$ were excited by the ion-sound instability driven by the current [6]. This article is devoted to the investigation of the complete structure of ion-sound turbulence. The auto-correlation coefficient of the ion-sound signal had the longliving "tail" up to $15-20 \%$. Two different auto-correlation times values were calculated: "short time" matches several periods of oscillations, and "long time" is about 100 periods of oscillations. Then the auto-correlation coefficient was calculated by means of reversed Wiener-Khinchin transform routine with the preliminary superposition of the spectra. This averaged coefficient value exceeds the value of the coefficient determined from the one-time realization (Fig.9(a)). For comparison, averaged auto-correlation coefficient of the numerical random signal is shown in Fig.9(b). The increase in the coefficient value is presumably due to long-living components in the ion-sound turbulent signal. The statistically steady spectrum includes several quasi-regular harmonics and background noise. These spectra were calculated earlier by differ-

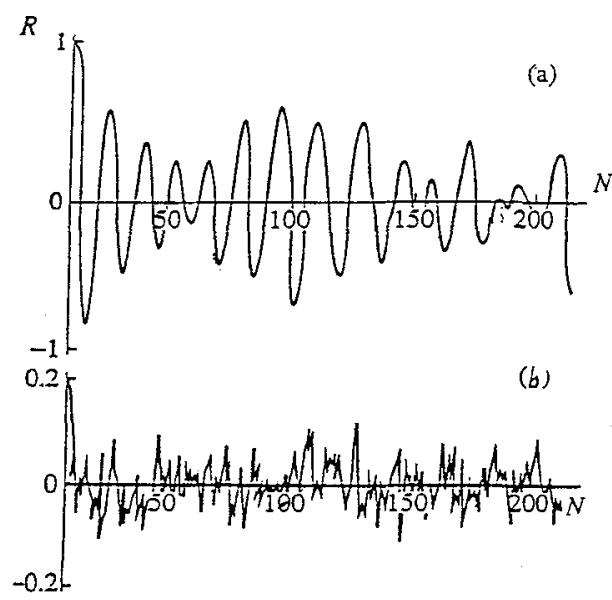

Figure 9: Averaged coefficients of autocorrelation. a) The ion-sound signal $(\triangle f=0.3-2.5 M H z)$. b) The numerical random signal.

ent numerical methods with statistical averaging $[\tau, 8]$.

Two power spectra were calculated from auto-correlation and cross-correlation functions of the signals from two probes located next to each other $\left(\Delta l \ll \lambda_{s}\right)$. When the signal can be represented as a superposition of the plane waves, then the two power spectra $P_{l+\Delta l, l}(w)$ and $P_{l, l}(w)$ (where $P_{l+\Delta l, l}(w)$ is the spectral density found from cross-correlation function, and $P_{l, l}(w)$ - is the spectral density found from auto-correlation function ) coincide, i.e. $P_{l+\Delta l, l}(w)=P_{l, l}(w)$. However the power spectra shown in Fig. 10 are different. This difference points out that the low-frequency signal in our experiment cannot be represented as superposition of plane waves.

The distribution of the averaged (20 realizations) phase over frequency harmonics in the ion-sound spectrum is shown in Fig.11. This distribution is completely occasional, that confirms the turbulent character of ion-sound oscillations, excited in the current driven plasma. 
We analyzed the probability distribution function (PDF) of the ion-sound turbulence. The curtseys of ion-sound PDF was $\varepsilon>3$ in the presence of drift regular modes, and it was $\varepsilon<3$ in the absence of drift. Two histograms shown in Fig.12 illustrate this result ( $a, b$ with and without drift, respectively). The third curve (c) is drawn for the gaussian probability distribution with $\varepsilon=3$. In the absence of drift, the PDF of ion-sound turbulence is close

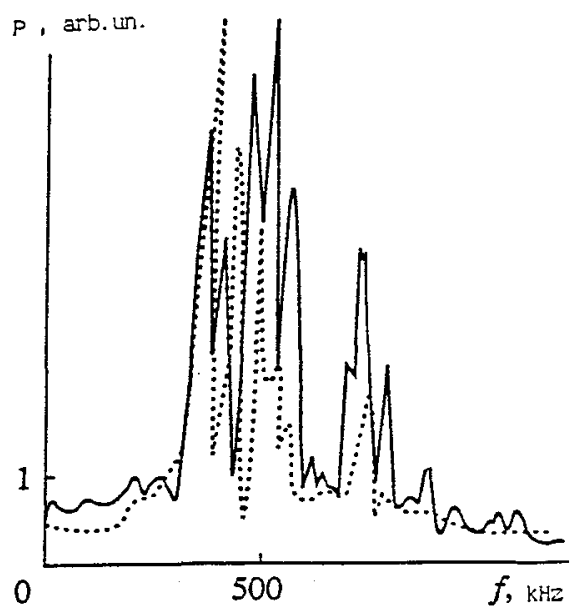

Figure 10: Power spectra of ionsound turbulence, calculated from: a) auto-correlation function; b) crosscorrelation function $(\Delta l=2 \mathrm{~mm})$.

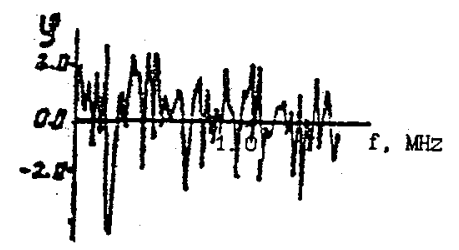

Figure 11: Averaged phase in the ionsound spectra. 20 realizations.

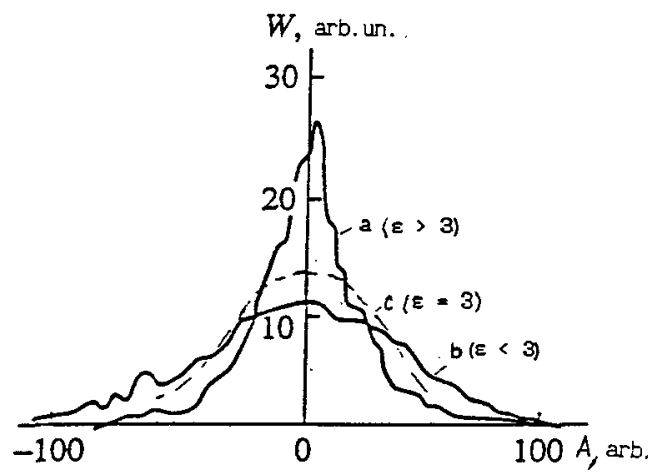

Figure 12: Histograms of ion-sound turbulent signals: a) with drift, b) without drift; c) Gaussian signal.

to the PDF of noise. In the presence of drift, the PDF points to more organized state. The possible relation of the ionsound turbulence with drift vortexes is discussed in [8].

We may conclude that this turbulence is the strong low-frequency turbulence. This turbulence is mixed with long-living quasi-linear signal of nonlinear nature. This strong ion-sound turbulence is excited due to the ionsound current instability. In the experiment the current value exceeds the current threshold of ion-sound instability. In this case, the ion-sound turbulence occurs at all frequencies over the ion-sound spectrum. When the growth of ion-sound instability occurs near the threshold, only several harmonics are excited with frequencies close to $\omega_{L i}$. Three-wave processes give rise to lowfrequency waves filling stationary spectra of weak ion-sound turbulence with other frequencies.

The use of bispectral analysis allows one to discriminate between waves spontaneously excited by the plasma and those generated by the former waves by the non-linear coupling [9]. 
Typical bicoherence spectra and integrated bicoherence are represented in Fig. $13(a, b)$. Only $1 \%$ of total power at frequency was coupled through threewave interactions. We conclude, that the waves harmonics of he strong ionsound turbulence were excited spontaneously all over the ion-sound spectrum.
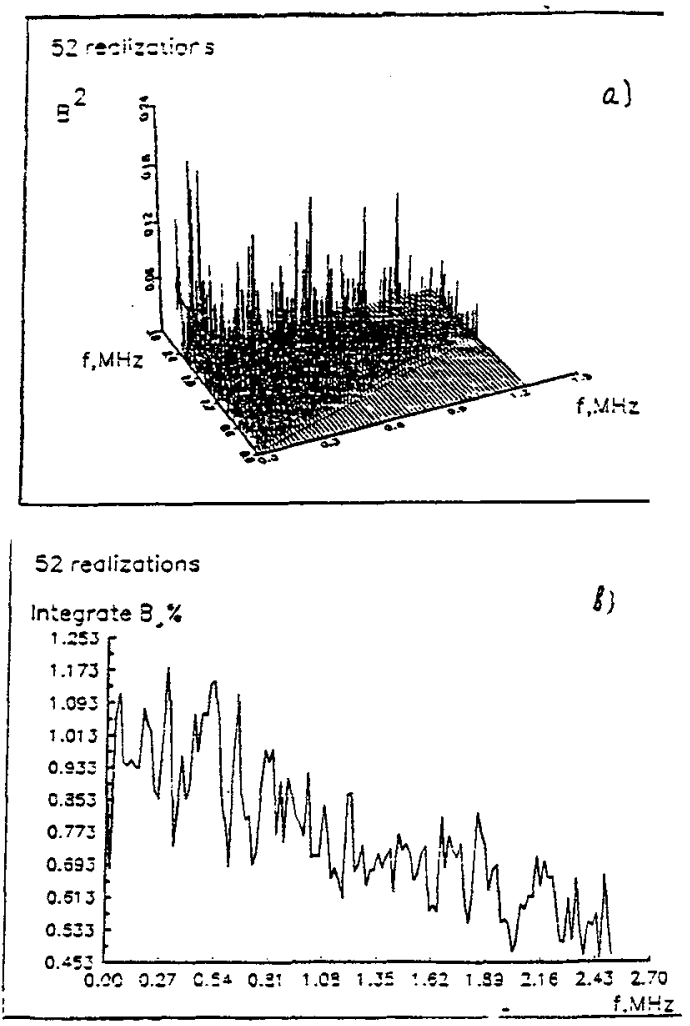

Figure 13: Bicoherence spectrum (a) and integrated bicoherence (b) of ionsound signal.

\section{References}

[1] A. G. Shkvarunets et al., Plasma Phys.20 (1994), 682-685

[2] V. P. Tarakanov, USER'S Manual for Code "KARAT", BRA, inc.V.A., USA (1992)

[3] M. A. Krasilnikov et al. JETP 108 (1995), 521-5:30

[4] G. M. Batanov et al. Proc. Lebedev Inst., Moscow, 160(1985),122134

[5] F. F. Asadullin et al. Plasma Phys., 7(1981) , 414-419

[6] G. M. Batanov et al. Preprint FIAN, Moscow, 53(1983),1-12

[i] V. V. Abrakov et al. XIX ICPIG, Belgrad ,1(1989),271-272

[8] V. V. Abrakov et al. Plasma Phys. 20(1994),1069-1076

[9] C. Hidalgo et al. Phys.Rev.Lett., $71(1993), 3127-3131$ 